\title{
Mathematical modeling for flocking flight of autonomous multi-UAV system, including environmental factors
}

\author{
Youngho Kwon', Jun Hwang2* \\ ${ }^{1,2}$ Dep. of Software Convergence, Seoul Women's University \\ [e-mail: yhkwon@swu.ac.kr, hjun@swu.ac.kr] \\ *Corresponding author: Jun Hwang
}

Received May 10, 2019; revised September 10, 2019; accepted November 9, 2019;

published February 29, 2020

\begin{abstract}
In this study, we propose a decentralized mathematical model for predictive control of a system of multi-autonomous unmanned aerial vehicles (UAVs), also known as drones. Being decentralized and autonomous implies that all members make their own decisions and fly depending on the dynamic information received from other unmanned aircraft in the area. We consider a variety of realistic characteristics, including time delay and communication locality. For this flocking flight, we do not possess control for central data processing or control over each UAV, as each UAV runs its collision avoidance algorithm by itself. The main contribution of this work is a mathematical model for stable group flight even in adverse weather conditions (e.g., heavy wind, rain, etc.) by adding Gaussian noise. Two of our proposed variance control algorithms are presented in this work. One is based on a simple biological imitation from statistical physical modeling, which mimics animal group behavior; the other is an algorithm for cooperatively tracking an object, which aligns the velocities of neighboring agents corresponding to each other. We demonstrate the stability of the control algorithm and its applicability in autonomous multi-drone systems using numerical simulations.
\end{abstract}

Keywords: Autonomous aerial vehicles, autonomous navigation, decentralized multicopter flock, self-propelled flocking, swarm flight

A preliminary version of this paper appears in Proceedings of the 10th International Conference on Internet (ICONI 2018), December 16-19, 2018, Phnom Penh, Cambodia.

This work was supported by a rearch grant from Seoul Women's University(2019) 


\section{Introduction}

$\mathbf{W}_{\text {ith their maneuverability and increasing affordability, autonomous unmanned aerial }}$ vehicles (UAVs) have received a growing interest in their ability to fly autonomously and automatically without human intervention. With the rapid technological advances made in electronics, interest is shifting toward collaborative UAV systems [1]. Although single-UAV systems are already in use, a single-UAV system cannot complete multi-faceted missions properly across large areas, due to battery constraints, self-provisioning, and other restrictions[2, 3]. A multi-UAV system can cover a wider area than a single UAV because the drones work in cooperation with each other. However, in addition to the advantages of running multiple drones, there exist numerous challenges and prominent design problems to be overcome before operation of a group of UAVs can be realized. Comparing multi-UAV systems with single-UAV systems, we find that one of the unique challenges of a multi-UAV system is communication[2-4]. Single-UAV systems use ground-based and satellite communication infrastructures but can also establish communication links between the drone and airborne control systems. In all cases, UAV communications are built between the UAV and the infrastructure [2, 3]. As the number of UAVs increases, effective network architecture design becomes a critical issue in preventing issues, such as broadcasting storms. Another challenge is group flight. If drones are to fly as a group[5-7] they must move in the same direction, and each must maintain proper spacing between itself and the others. A drone flying in an incorrect direction is sufficient to bring about a risk of collision with one of its neighbors, and if the distance between the drones is too large, one or more of the drones could leave the group due to communication failure. These technical issues arise from the partially or entirely autonomous execution of all necessary flight processes, such as stable hovering, flying in the specified direction at the encoding speed, or adjusting sufficiently quickly to time-dependent altitude and flight direction requirements. Additionally, for a group of autonomous robots, communication between the robots usually has a limited range[5]. Similarly, a drone can only send messages (e.g., its location and speed) to its nearest neighbors.

The most straightforward agent-based system of flocking designates the arrangement as satisfying a mathematical axiom: Each unit adjusts its velocity vector closer towards the average velocity vector of its neighbors (including its own)[8]. This localization is the essential characteristic of the flocking model in herd behavior. The unit adjusts its speed to match the lowest speed of the other units, though only within a limited range. In a flocking model, the vectors of each node develop individually through a dynamic system. This similarity allows some principles of animal herd behavior models to be incorporated into the control dynamics of autonomous robots[9]. Because each of the robots in the self-flying group has an onboard computer and sensor, control dynamics are discrete and distributed. A system allowing for flexible collective action should have the ability to securely handle response time and communication delays caused by noise input or spontaneous environmental changes. These can still have a destabilizing impact on robotic systems.

The main contribution of this work is a simple decentralized general mathematical model for predictive control of multiple autonomous UAVs. We consider a realistic scenario through the addition of Gaussian noise to describe such factors as time delay due to inaccuracies of the onboard sensors, and inter-drone communication delay due to the environment. In the mathematical analysis and numerical simulation, we demonstrate that the grouping of agents into a flock within a finite time can be assured by boundary 
conditions under a specific uniform common connectivity condition, and further that the agents asymptotically congregate towards an inflexible and uncongested group flight.

The remainder of the paper is organized as follows. In Section 2 we present relevant previous work. The mathematical algorithms for formulating the flocking problem and some preliminary concepts are introduced in Section 3. In Section 4, The numerical experiments are performed and performance evaluation results are presented along with comparisons with the multiple implicit leaders aided schemes proposed in [32]. Finally, in section 5, we conclude our paper and indicate future work to be done.

Throughout this paper, we use $\overrightarrow{n_{i j}}$ for vector and $\bullet$ for vector inner product. $R_{i j}$ and $d$ are sensing range and desired distance, respectively.

\section{Related Work}

The cooperative movement of autonomously flying drones is one of the most recent problems to emerge in the study of complex dynamic systems. The joint movement of individuals is a common phenomenon that can be detected in conventional biological systems such as large groups, mammal migrations, and cell movements. These systems display some common characteristics $[9,10]$. The speed vectors of adjacent individuals tend towards each other by matching each other's load share and energy consumption. According to Reynolds [11], there are three key heuristic rules: separation at short-range to avoid collisions, local interactions (alignment rules) for aligning velocity vectors and long-range attraction of individuals to keep the group flight together. These rules can be interpreted mathematically in agent-based models, which are discrete or continuous dynamic systems that describe the time variation of the speed of individual units [7, 12].

Over the years there have been many studies based around Reynolds' three rules. R. OlfatiSaber et al. considered agreement algorithms in full generality, they put forwards ideal results for the convergence properties of agreement algorithms and conducted a numeral investigation. H.G. Tanner et al. presented a simple parameter algorithm to investigate the agreements (especially velocity matching) of models rather than actual models using graph theory. In [13] a local attractive/repulsive function is proposed in a separate time environment, to manage separation and consistency issues. Artificial potentials and rotating mechanism forces are designed for obstacle avoidance in $[14,15]$. Lately, we have seen additional break-throughs being made for Reynolds' blocking models, including the stable blocking analysis of $[14,16,17]$. In [18], Tanner et al. proposed a globally stable multi-agent interaction model, which was applied to systems with point mass dynamics. The $\alpha$-lattice model was proposed as a theoretically calculated structure with an added random duplication effect for flocking $[19,20]$.

Various works have considered a reduced objective in flocking problems, where $N$ nonholonomic agents moving at unit speed must align their velocity vectors. Moshtagh et al. [21] presented a vision-based flocking control to enhance the existing control laws enabling a group of individuals to align with the leading orientation, as long as the proximity-based closed-graph representing the community topology is connected. In a similar method, Paley et al. [7, 22] assume an all-to-all communication topology and advance both the control laws that synchronize the group's cooperative movement and the topology of the entire communication infrastructure, that is, by aligning the agent orientation in a known flow area (e.g., a constant velocity wind). While the previous two papers treat objects such as unit velocity vectors from an engineering perspective with first-order rotational dynamics, Mellish et al. [7] 
extended their work by using a backstepping approach to synchronize the second-order rotational dynamics and mass movements in unit space for unmanned vehicles in a uniform flow area.

Many studies have considered using sensors for the autonomous flight of drones. Global Positioning System (GPS) based autonomous drone swarm models are proposed [12, 17, 23, 24]. These systems typically have much smaller cluster sizes than pre-programmed drones, due to inter-GPS signal interference. Except for [24], where 50 fixed-wing UAVs were flown and operated at different altitudes without a sensor-based collision avoidance device, the GPS-based outdoor herds proposed in these studies were composed of less than ten drones.

Other studies used sensors to measure the environment, to enable drones to navigate through an unknown environment with relatively low operating costs and high flexibility. However, it is difficult to achieve the accuracy of a model that describes the target, or one with prior knowledge of the environment $[5,6]$. In order to solve the problems caused by inaccuracies in the representation of dones' surrounding environments, reinforcement learning (RL) has been proposed. Several papers focus on realizing trajectory tracking/hovering, by applying the RL algorithm to UAV control. Faust et al. [25] have proposed a motion plan for UAVs with stationary loads, generating traces with minimal residual vibration by employing a framework with an RL feature. Bou-Ammar et al. [26] used an adaptive iterative RL algorithm, comparable to a model-based feedback linearization controller, to obtain a stable trajectory in UAV maneuvers. Santos et al. [27] proposed an RL-based automation method to allow parameter tuning of a PID (Proportional, Integral, Derivative) controller for UAVs even under adverse weather conditions.

\section{PROBLEM FORMULATION AND PRELIMINARIES}

\subsection{Problem statement and formulation}

We need to distribute the minimum amount of UAVs capable of providing coverage, by setting the UAVs at locations where each UAV will offer complete coverage. In order to do that, the drones can be positioned based totally on knowledge of geographical locations, which every node can outline using the GPS. However, it is challenging to track all GPS signals because every drone has a different signal intensity. Furthermore, if the distance between the drones is too small, it becomes difficult to determine the exact number of drones, due to the interference of signals. In this problem, we assume: (1) A time-slotted system with a total duration of $T$ and one time per slot (2) That each UAV covers a given area for one or multiple time slots. (3) That each UAV can move (from location $x$ to location $y$ ) or (from location $x$ to $x^{\prime}$ ), with this process taking up a one-time slot.

Through these, we assume in the rest of the paper that the drones make decisions at each interval of time as follows:

1. Each UAV is represented as a node, $N=\{1,2,3 \cdots, n\}$ where $n$ is the total number of UAVs in the fleet, and all nodes have the same functions.

2. A UAV can connect to any unmanned aircraft using one-hop communication.

3. Sensors built onto UAVs are operated periodically to identify targets around them, and the power consumed by these sensors is uniform.

4. The UAVs are flying at constant altitudes and speeds, and there are external factors that interfere with flight (e.g., wind, noise and moisture). 
As a result of flocking, each member of the group is directed in the same direction as the other members, and the members of the group maintain a constant distance from each other to keep the Reynolds' flocking three rules [12]. For this purpose, the average size of grouping or clustering should take into account the structural dimension of each individual's connection to forming the group. Otherwise, either too weak or too strong signals could attract disproportionate resources on a single target, thus interfering progressive development of the flocking behavior. As an effect of stable positioning, Other drones can identify possible targets through repeated detection and can look around the detected location. it is necessary to know exactly where the drone is located at all times. The relative positioning of the UAV is also usually determined by the GPS signal. During flock flights, it is a difficult problem of classifying individual GPS signals over the times among drones. In this study, we considered the method to determine the drones' location without using GPS.

To determine the number of drones in a given area without using a GPS signal, we follow the model presented in [20] under free-flow traffic conditions. We assume that UAVs within the communication range $R$ are included in a segment of length $l$ (meters). Also, the UAVs within range assume that each time they receive a message successfully, they know the current status of the other UAVs. The communication between UAVs is considered to be one-dimensional (1D) in the length segment and the number of UAVs is Poisson distributed as the parameter $\rho$ (flight aircraft density), the probability $P(x, \rho)$ of finding $x$ UAVs in a length $l$ is given as follows:

$$
P(x, l)=\frac{(\rho l)^{x}}{x !} e^{-\rho l}
$$

Under those conditions, each unmanned aircraft is programmed to maintain a constant speed while navigating along a given segment of $l$. During the group flight, the time dependency of the ith unit's desired velocity can be written as a function of the other units' positions $(x)$ and velocities $(v)$ :

$$
v_{i}^{d}=\sum_{i, j=1}^{N} R_{i j}\left\{x_{i}(t), v_{j}(t)\right\}, i, j \in N,
$$

where $N$ is the number of agents and $R_{i j}$ is a function containing arbitrary features of the controlling dynamics. Since this paper assumes that the UAVs fly at a fixed altitude, the lift and drag coefficients that vary depending on the speed of the aircraft are constant.

In the ideal case, the velocity of the $i$ th drone changes immediately to $v_{i}^{d}$ at time $t$. To model UAV herd behavior, we propose in novel mathematical terms that each UAV observes its nearest neighbors, as illustrated in Fig. 1. With the proposed model, a group of UAVs has information from each neighboring drone and will cooperatively fly towards target points. 


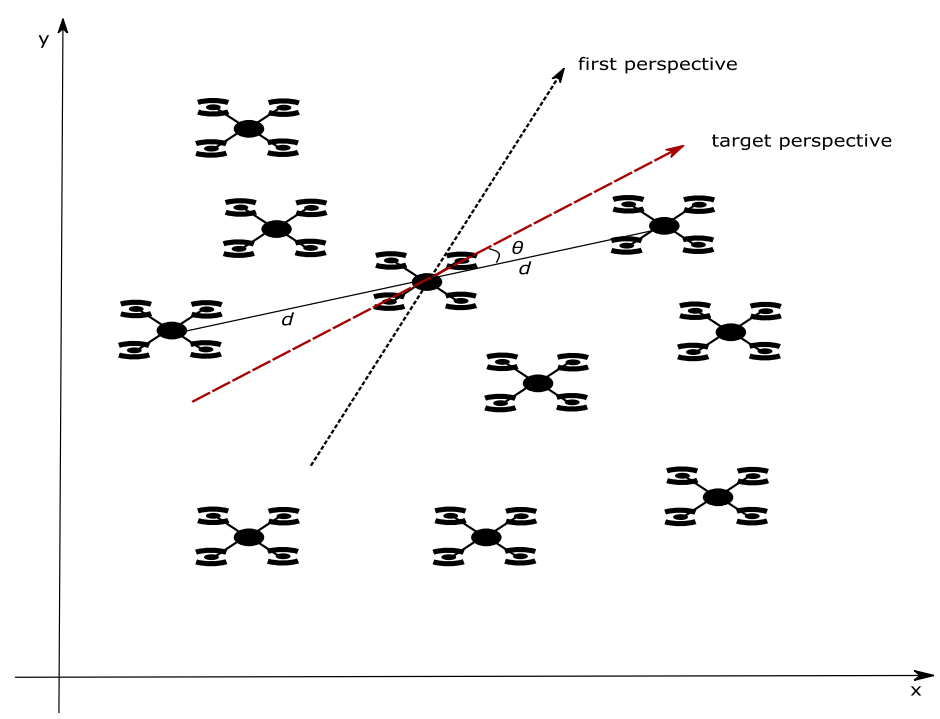

Fig. 1. Schematic design of a UAV's state space

However, as $R_{i j}$ is never ideally functional; some of its deficiencies shall be modeled:

(i) Grouping occurs at a finite time $T$ over $[T,+\infty]$

(ii) The response time and agility of the UAVs are determined by the refresh rate of the sensor input data. We reflect a constrained refresh rate of the sensors; every unit updates the sensor's data value with a frequency $t_{s}^{-1}$. In our proposed model, $t_{s}^{-1}$ is constant.

(iii) The UAV-to-UAV communication takes place in a finite range $d$, as shown in Fig. 1. If two UAVs are too far from each other, they cannot exchange messages. For modeling this effect, a maximum communication range $c_{r}$ is defined in our approach. Consequently, if the distance between two UAVs is greater than $c_{r}$, they cannot communicate with each other. In other words: the $R_{i j}$ function depends on $x_{j}$ only if $\left|x_{j}(t)-x_{i}(t)\right|=d$. This can be written in the following vector formats:

$$
x_{j}(t)-x_{i}(t) \rightarrow d \cdot \vec{n}_{i j}(t), \quad t \rightarrow \infty, i, j \in N
$$

Here $\vec{n}_{i j}$ is the unit vector representing the direction from $\mathrm{UAV}_{i}$ to $\mathrm{UAV}_{j}$. Due to the mass, aerodynamic effects, and certain features of the low-level control algorithm, the flying UAV cannot change its velocity immediately. Therefore, the initial distance between UAVs and their interaction range must be far enough, and the initial velocity should also be smaller so as to meet the requirements of $\left|x_{j}(t)-x_{i}(t)\right|=d$, which guarantees the collision avoidance and stability of the system. This vector can be represented in two different ways, as shown in the following equation:

$$
\vec{n}_{i j}=\frac{x_{j}-x_{i}}{\left\|x_{j}-x_{i}\right\|}, \vec{n}_{i j}=\left[\cos \theta_{i j} \sin \theta_{i j}\right]^{T}
$$


where $\theta_{\mathrm{ij}}$ is the orientation angle. In this study, We suppose that the minimum distance considering flight direction between UAVs are always greater than zero, which reveals that collision avoid-ance among vehicles is achieved successfully.

In formulating the grouping problem with a velocity vector, we oppose finding a decentralized algorithm for each agent so that group action occurs. To satisfy this, we had to set up a specific connectivity condition for grouping. A typical condition is based on the early models of animal swarms [12, 18, 28, 29]. We have followed [5, 9, 11] in saying that 'self-organization' in this paper means that individuals reach a well-defined group status according to their own decisions. The agent's desired speed is now the sum of interactions with other unmanned aircraft and defines the self-propelled behavior and interactions within the restricted area. Under the above conditions, we define the UAVs as self-propelled automatic particles with ideal flock velocity in section 3.2.

\subsection{Mobile strategies of UAVs}

This section introduces the processes that allow a large group of individual UAVs to navigate from their original location to their destination. In this paper, we assume that there are no obstacles in the path that the drones take towards their destination, and that the destination to be moved towards is stored in the memory of the drone after being entered in advance by the user.

The control strategy used by drones to navigate is similar to the general strategy used to perform regular flights. This strategy consists of forming a navigation vector that includes the location and therefore the direction of the destination. First of all, we define the drones as autonomous with a preferred velocity $v_{\text {flock }}$,

$$
v_{x, y, n}=v_{\text {flock }} \frac{\left|v_{i}\right|}{\left|v_{j}\right|} \text {, }
$$

where $v_{x, n}$ is the longitudinal speed at time slot $t_{i}$ of each UAV and $v_{y, n}$ the latitudinal speed at the time slot $t_{i}$ of each UAV. They are the constant flocking speed rates that the units try to maintain. To avoid collisions, we define a local linear repulsion between the units as their (direct) connectivity intensity over $\left[t_{i}, t_{i+1}\right)$. This paper assumes that each UAV receives information identifying the condition of all the drones in its range of sight, then determines its behavior with that information. For any two agents $i, j$ in the UAV-node model, we define the distance selection process as follows:

Step1: Assuming that the drones know the coordinates of each drone, the center of gravity of the region can be calculated. If $\left(X_{c}, Y_{c}\right)$ is the center of gravity in a given area, which coordinates should be found using the least-squares method, selecting the point which minimizes the average distance to all drones in the area. The concrete calculation method is as follows:

$$
\left(X_{c}, Y_{c}\right)=\min \sum_{i=1}^{N}\left[\left(x-x_{i}\right)^{2}+\left(y-y_{i}\right)^{2}\right],
$$

where $\left(x_{i}, y_{i}\right)$ are the coordinates of each UAV;

Step 2: The distance of the UAV from the center of gravity for the group is satisfied with the following: 


$$
d\left(t_{i}, t_{i+1}\right)=\min _{0 \leq E \leq 1} E \cdot d_{i j}\left(t_{i}, t_{i+1}\right)
$$

where $d_{i j}$ is $\sqrt{\left(x_{c}-x_{i}\right)^{2}+\left(y_{c}-y_{i}\right)^{2}}$ and $E$ represents Gaussian noise. This timedependent Gaussian noise term models fluctuating ecological effects such as windward inconsistencies of the low-level control algorithm. In this paper, we also consider the amplitude of fluctuations in the measured position due to internal noise to be in the same range.

Step 3: Calculate the distance between drones in the radius.

The key principle of collective action is a form of global positioning constraint, that contributes to the reliability of the group. In the simulation, the properties of positioning constraints can be replaced using periodic boundary conditions. To study the herd model through simulation, we used the following as the boundary condition equations for collective flight [30, 31]:

$$
s(x, R, d)=\left\{\begin{array}{ccc}
0 & \text { if } & x \in[0, R] \\
\frac{1}{2}\left(\sin \left(\frac{\pi}{d}(x-R)\right)+1\right) & \text { if } & x \in[R, R+d] \\
1 & \text { if } & x>R+d
\end{array}\right\}
$$

where $s(x, R, d)$ is a sigmoid curve that gently reduces the strength of the repulsion inside the area.

To determine the possibilities of various actuator configurations, and to benchmark the collision avoidance path and speed controller, we use simplified multiple UAV models to formulate and solve optimal control problems. For this, the dynamics of the UAV are modeled using point masses (particle model). By using the Lyapunov-based approach [29], We assume $A \in R^{n x n}, P=P^{T} \in R^{n x n}$. It follows that $Q=Q^{T} \in R^{n x n}$. If for $\dot{x}=$ $A x, V(z)=z^{T} P z$ continuous-linear time, we have $\dot{V}(z)=-z^{T} Q z$, where $P, Q$ satisfy the state vector variable written as:

$$
Z=\left[\begin{array}{llllll}
X & Y & V_{x} & V_{y} & R_{x} & R_{y}
\end{array}\right]^{T}
$$

To simplify the result of the equation of motion and flight model of a system, we form a system of first-order differential equations in the main space form as below;

$$
Z^{\prime}=\left[\begin{array}{c}
X \\
Y \\
V_{x} \\
V_{y} \\
R_{x} \\
R_{y}
\end{array}\right]=\left[\begin{array}{c}
V_{x} \\
V_{y} \\
(x \cos \theta-y \sin \theta) / m \\
(x \cos \theta+y \sin \theta) / m \\
v_{x, n} / d_{x} \\
v_{y, n} / d_{y}
\end{array}\right]
$$


Where $m$ is the total mass of the UAV. Since we assume that UAVs fly at a constant speed and flocking flight in the interval $\left[t_{i}, t_{i+1}\right)$, the magnitude of the additive and multiplicative operations can be estimated by using the least-squares error method. In the form of a leastsquares regression, the control input and estimated fault magnitude can be defined as:

$$
\vec{y}=\alpha+\beta \vec{u},
$$

where $\alpha$ and $\beta$ represent the magnitude of the additive and multiplicative faults, respectively. At least two related independent inputs and outputs should be required since $\alpha$ and $\beta$ are unknown parameters. With the data relationship pertaining to the historical time of input and output, $\alpha$ and $\beta$ can be estimated as follows, according to [30].

$$
\begin{gathered}
\alpha=\frac{\left(\sum_{j=1}^{M} x_{j}\right)\left(\sum_{j=1}^{M} y_{j}\right)-\left(\sum_{j=1}^{M} x_{j}\right)\left(\sum_{j=1}^{M} x_{j} y_{j}\right)}{M\left(\sum_{j=1}^{M} x_{j}^{2}\right)-\left(\sum_{j=1}^{M} x_{j}\right)^{2}} \\
\beta=\frac{\left(\sum_{j=1}^{M} x_{j} y_{j}\right)-\left(\sum_{j=1}^{M} x_{j}\right)\left(\sum_{j=1}^{M} y_{j}\right)}{M\left(\sum_{j=1}^{M} x_{j}^{2}\right)-\left(\sum_{j=1}^{M} x_{j}\right)^{2}},
\end{gathered}
$$

where $M$ is the length of the drone's moving distance.

Let $F(z)$ denote the state of the general system. The aim is to minimize the objective function $F(z)$, which is defined as

$$
F(z)=\alpha z(0)+\beta z(T)+\int_{0}^{T} z^{T} Q d t .
$$

where $Q$ is the Gaussian antenna gain. Eqs. (9)-(14) form the optimal control problem and boundary conditions.

To find the optimum UAVs' location to satisfy a given equation, we can calculate using a variety of algorithms such as ant colony optimization, bee colony optimization, genetic algorithm, and particle swarm optimization. We performed a Matlab computer simulation by applying a particle swarm optimization to enhance the placement of UAVs for constants $\alpha$ and $\beta$ in the range [0,1], defined by the strength of the relative velocity components associated with $v_{0}$ at a maximal tracking speed.

\section{Numerical Results and Analysis}

In this section, we perform a mathematical simulation to show the usefulness of the algorithm proposed in section 3. Note that our goal is to show that the proposed mathematical model has stability through the simulation experiment. Therefore, the other parameters $\left(v_{o}, \alpha, \beta\right.$ and, $\left.d\right)$ are set to fixed default values. We have optimized our parameter choices to reliably complete target tracking with a smooth transition. 
To initialize the flocking state, we placed the UAVs within a square space of $5 \times 5$ kilometers $(\mathrm{km})$. The distance between drones is $d$ meters $(\mathrm{m})$. Ideally, after starting the simulation, the velocity vector of the UAV-nodes should be parallel and should have the same magnitude. The initial values are randomly selected from $[0,2 \pi)$ and $[0,0.3]$, respectively. We adopt the so-called random waypoint (RWP) model, in which nodes move immediately along line segments from one waypoint to the next. Based on RWP, the drones capture the random movement of other drones, such that they can imitate the movement of a flying UAV. After the simulation starts, the flight node moves in one direction at a constant speed for some time, and we track it immediately after that. After a certain period, the node randomly selects a new direction with a different speed and maintains it. Some relevant network parameters are listed in Table 1.

Table 1. Simulation parameters

\begin{tabular}{|c|c|c|}
\hline Parameter & Description (unit measure) & Set value \\
\hline \hline$N$ & Number of drones & 100 \\
\hline$B_{d}$ & Initial energy $(\mathrm{J})$ & 0.5 \\
\hline$m$ & Drone weight $(\mathrm{kg})$ & 1 \\
\hline$v_{0}$ & Preferred velocity far from the target position $(\mathrm{m} / \mathrm{s})$ & 2 \\
\hline$c_{r}$ & Characteristic size of the target area $(\mathrm{km})$ & {$[0,5]$} \\
\hline$d$ & Characteristic size of the “transition” area $(\mathrm{m})$ & $\{2,4,6\}$ \\
\hline$E$ & Delta-correlated (Gaussian) outer noise $\left(\mathrm{m}^{2} / \mathrm{s}^{2}\right)$ & $N(0,1)$ \\
\hline
\end{tabular}

Recently, the schemes in [32] have been published and introduced unique challenges for UAV swarm formation control protocols. As mentioned earlier, we compare the performance of the proposed scheme with these existing schemes to confirm the superiority of the proposed approach

Fig. 2 shows the effect of the total time required to reach the target point if a time delay element occurs in the system. If the characteristics of drones' swarm behavior are done under the Reynolds' three rule of flocking [11]. With alignment rules, drones tend to move in the same direction as nearby drones. and, the drone keeps a minimum distance able to provide the drone with flexibility and for a better exploration when they are flocking. With cohesion rules, the drone tends to move towards the flocking. According to Fig. 2, the additional Gaussian noise terms can also increase the instability caused by time delays in the flight state. In general, random noise usually works in inverse synchrony and quality, as it is a common feature of a delayed dynamic system. This result shows that as the number of UAVs increases, the signal is more vulnerable and the noise increases as the distance between UAVs becomes smaller. For a low signal-to-interference-plus-noise ratio (SINR) threshold however, the time taken to reach the target point appears to increase monotonically. One limitation of increasing density, especially when there is a time delay in the system, is that the total time required to reach the target point increases.

Fig. 3. shows the number of active drones following the proposed flocking algorithm as time increases. The drone operates by taking its power supply from the internal battery and maneuvers up and down as well as to the right and left to avoid obstacles (e.g., buildings) based on alignment, separation, and cohesion. Therefore, it is required to verify how long the flocking flight is maintained with limited power. For realistic simulation, we have chosen a model in which energy decreases over time $T$ according to Eqs. (3)-(7). For battery consumption, we randomly changed the direction of the drones in this simulation. 
In Fig. 3, with small $c_{r}$ values, each UAV-node updates information from neighboring UAVs independently of each other, thus each unit calculates their velocity on the same line of speed towards the target. However, increased noise between UAV-nodes may cause a collision. The shorter the distance between the drones, the greater the delay in sharing correct information of each drone and the more battery power consumed in updating the velocity vector. The experimental results show that the number of drones in flocking flight can be seen to drastically decrease as the value of $d$ decreases. If the finite range $d$ increases close to the noise-avoiding range, a cooperative flocking state can be achieved and last a given simulation time.

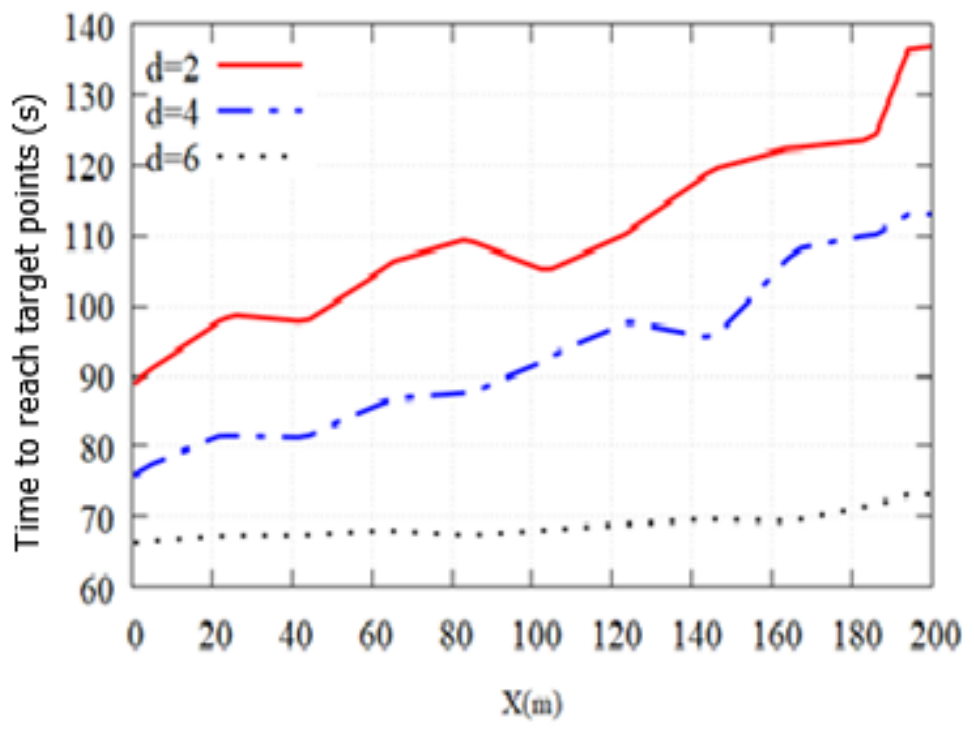

Fig. 2. Time to reach target point with $v_{0}=2 \mathrm{~m} / \mathrm{s}, \alpha=\beta=0.5, m=1 \mathrm{~kg}$ and drones $=100$

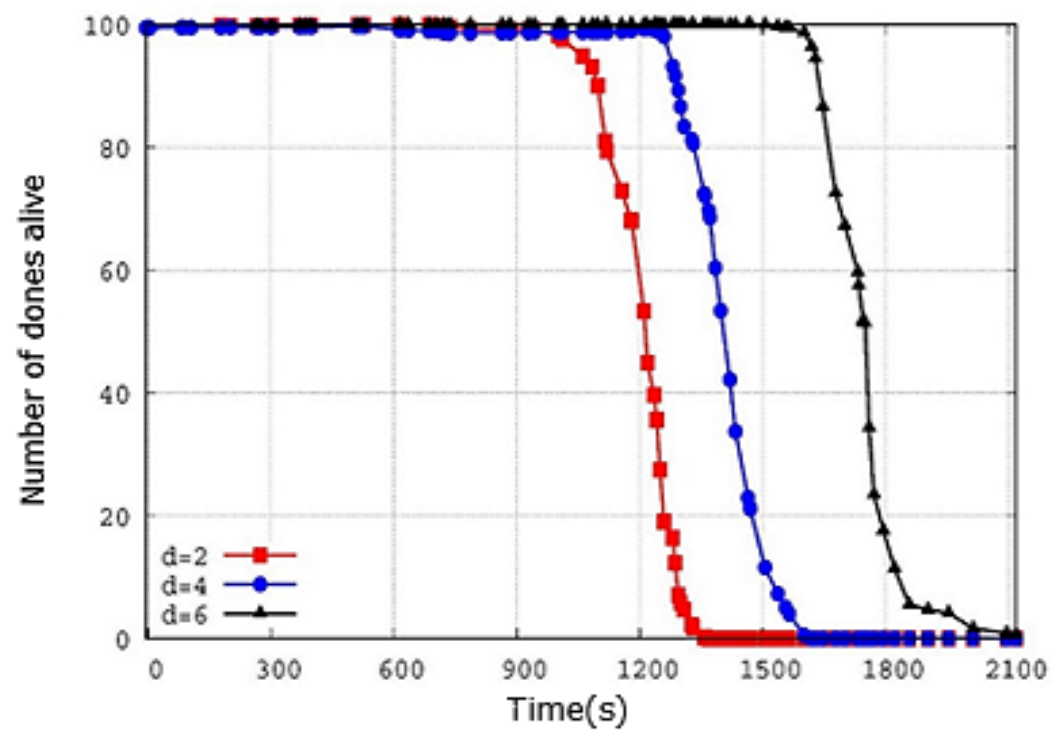

Fig. 3. Comparison of flocking duration time 
Fig. 4 shows the performance comparison for trajectories of UAVs, note that the axes scale of $\mathrm{X}$ and $\mathrm{Y}$ are different. We compared it with the proposed method using the path measurement and error rate proposed by [32]. In this paper, the grouping and flight direction of drones is determined by a linear equation based on the Lyapunov-based method. The method proposed in this paper does not require the selection of leaders around to determine the direction of flight [32]. When the trajectory is low $(x<0.15)$, the performance of all the schemes has similar trends. As the traffic intensity increases, the proposed scheme has better flocking behavior than that of [32].

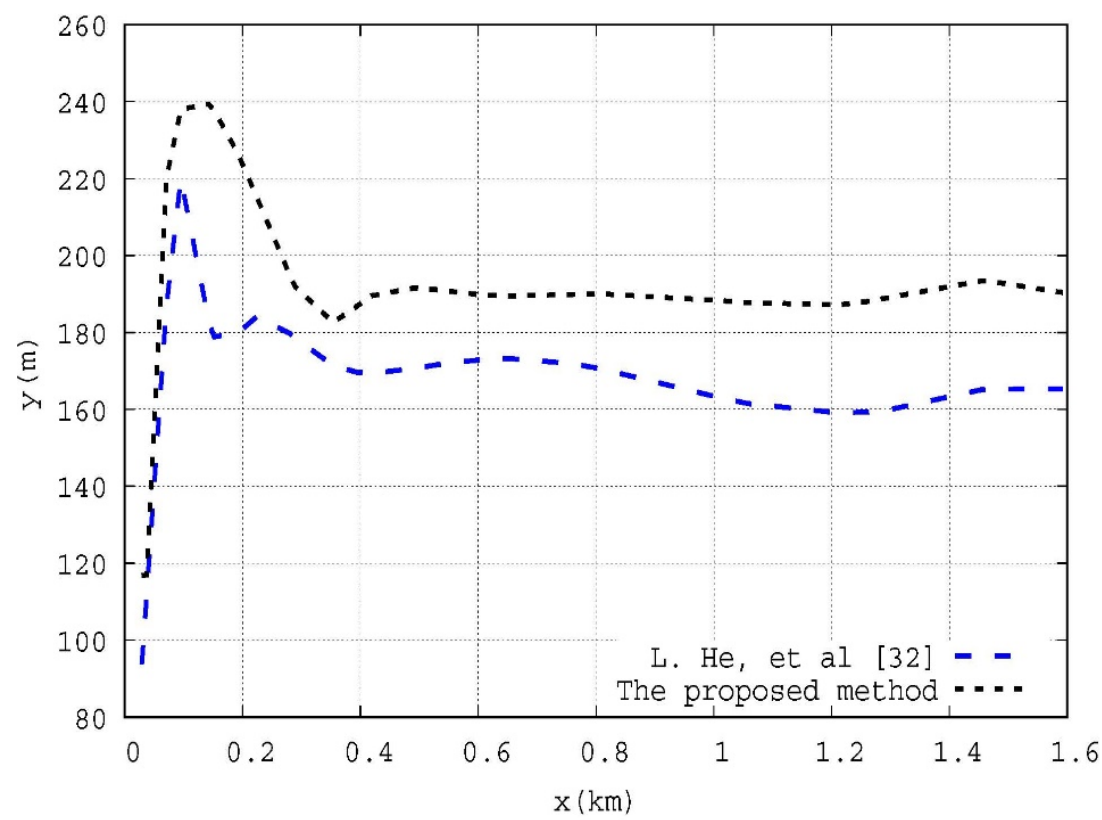

Fig. 4. Trajectories of UAVs

\section{Conclusion}

In this study, we propose a mathematical model for the development of decentralized control algorithms for autonomous multi-UAV flocking. By using the Lyapunov-based approach, we present uniform common conditions to ensure speed synchronization and collision avoidance for a crowded flock. The proposed mathematical model demonstrated good performance in simulations based on alignment, separation, and cohesion under the Reynolds' three rules.

Implementing autonomous collision avoidance without using a centralized method for a large scale synchronized cluster flight in a restricted area is a problem that has not yet been solved. We have demonstrated the possibility of autonomous flight capable of avoiding collisions in the boundary area through numerical simulations with 100 autonomous quadcopters. For this, we used an extensible, optimized mathematical expression based on the explicit processing of faithful, dynamic modeling and the motion constraints of the crowded equation. Results on numerical simulation prove the benefits of flocking, in terms of the effectiveness of the limited battery power. 
If the structural parameters are correctly adjusted under a given scenario, the entire mechanism can be used more effectively. Thus, It is desirable to adjust the parameters for a specific search area. Another critical issue is the implementation and testing. Usually, the theoretical results are verified through computer simulations; however, for practical purposes, this may not be sufficient. Therefore, extensive experimental research is needed in this field. In the future, we aim to expand our current research to study more realistic UAV-to-UAV communication and study a target location system in which nodes autonomously adjust their location.

\section{References}

[1] L. Gupta, R. Jain, and G. Vaszkun, "Survey of important issues in UAV communication networks," IEEE Communications Surveys \& Tutorials, vol. 18, no. 2, pp. 1123-1152, 2015. Article (CrossRef Link)

[2] H. Jiang, Z. Zhang, and G. Gui, "Three-dimensional non-stationary wideband geometrybased UAV channel model for A2G communication environments," IEEE Access, vol. 7, pp. 26116-26122, 2019. Article (CrossRef Link)

[3] H. Jiang, Z. Zhang, L. Wu, and J. Dang, "Three-dimensional geometry-based UAV-MIMO channel modeling for A2G communication environments," IEEE Communications Letters, vol. 22, no. 7, pp. 1438-1441, 2018. Article (CrossRef Link)

[4] H.-s. Kim and Y. Kim, "Trajectory optimization for unmanned aerial vehicle formation reconfiguration," Engineering Optimization, vol. 46, no. 1, pp. 84-106, 2014.

Article (CrossRef Link)

[5] H. M. La, "Multi-robot swarm for cooperative scalar field mapping," Handbook of Research on Design, Control, and Modeling of Swarm Robotics: IGI Global, pp. 383-395, 2016.

Article (CrossRef Link)

[6] H. M. La, W. Sheng, and J. Chen, "Cooperative and active sensing in mobile sensor networks for scalar field mapping," IEEE Transactions on Systems, Man and Cybernetics: Systems, vol. 45, no. 1, pp. 1-12, 2015. Article (CrossRef Link)

[7] R. Mellish and D. A. Paley, "Backstepping control design for motion coordination of selfpropelled vehicles," in Proc. of 49th IEEE Conference on Decision and Control (CDC), pp. 5468-5473, 2010. Article (CrossRef Link)

[8] T. Vicsek, A. Czirok, E. Ben-Jacob, I. I. Cohen, and O. Shochet, "Novel type of phase transition in a system of self-driven particles," Phys Rev Lett, vol. 75, no. 6, pp. 1226-1229, Aug 7, 1995. Article (CrossRef Link)

[9] M. Brambilla, E. Ferrante, M. Birattari, and M. J. S. I. Dorigo, "Swarm robotics: a review from the swarm engineering perspective," vol. 7, no. 1, pp. 1-41, 2013.

Article (CrossRef Link)

[10] T. Vicsek and A. Zafeiris, "Collective motion," Physics reports, vol. 517, no. 3-4, pp. 71140, 2012. Article (CrossRef Link)

[11] C. W. Reynolds, "Flocks, herds and schools: A distributed behavioral model," ACM SIGGRAPH computer graphics, vol. 21, no. 4, pp. 25-34, 1987. Article (CrossRef Link)

[12] S. Hauert, S. Leven, M. Varga, F. Ruini, A. Cangelosi, J.-C. Zufferey, and D. Floreano, "Reynolds flocking in reality with fixed-wing robots: communication range vs. maximum turning rate," in Proc. of 2011 IEEE/RSJ International Conference on Intelligent Robots and Systems, pp. 5015-5020, 2011.

[13] W. Ren and R. W. Beard, "Consensus seeking in multiagent systems under dynamically changing interaction topologies," IEEE Transactions on automatic control, vol. 50, no. 5, pp. 655-661, 2005. Article (CrossRef Link) 
[14] V. Gazi and K. M. Passino, "Stability analysis of swarms," in Proc. of the 2002 American Control Conference (IEEE Cat. No. CH37301), vol. 3, pp. 1813-1818, 2002.

Article (CrossRef Link)

[15] O. Khatib, "Real-time obstacle avoidance for manipulators and mobile robots," in Proc. of 1985 IEEE International Conference on Robotics and Automation, vol. 2, pp. 500-505, 1985. Article (CrossRef Link)

[16] D. E. Chang, S. C. Shadden, J. E. Marsden, and R. Olfati-Saber, "Collision avoidance for multiple agent systems," in Proc. of the 42nd IEEE Conference on Decision and Control, Maui, HI, USA, pp. 539-543, 2003. Article (CrossRef Link)

[17] R. G. Braga, R. C. Da Silva, A. C. Ramos, and F. Mora-Camino, "Collision avoidance based on Reynolds rules: A case study using quadrotors," Information Technology-New Generations: Springer, pp. 773-780, 2018. Article (CrossRef Link)

[18] H. G. Tanner, A. Jadbabaie, and G. J. Pappas, "Stable flocking of mobile agents, Part I: Fixed topology," in Proc. of 42nd IEEE International Conference on Decision and Control (IEEE Cat. No. 03CH37475), vol. 2, pp. 2010-2015, 2003. Article (CrossRef Link)

[19] R. Olfati-Saber, "Flocking for multi-agent dynamic systems: Algorithms and theory," 2004.

[20] L. Moreau, "Stability of multiagent systems with time-dependent communication links," IEEE Transactions on automatic control, vol. 50, no. 2, pp. 169-182, 2005.

Article (CrossRef Link)

[21] N. Moshtagh and A. Jadbabaie, "Distributed geodesic control laws for flocking of nonholonomic agents," IEEE Transactions on Automatic Control, vol. 52, no. 4, pp. 681-686, 2007. Article (CrossRef Link)

[22] D. A. Paley and C. Peterson, "Stabilization of collective motion in a time-invariant flowfield," Journal of Guidance, Control and Dynamics, vol. 32, no. 3, pp. 771-779, 2009.

Article (CrossRef Link)

[23] G. Vásárhelyi, C. Virágh, G. Somorjai, N. Tarcai, T. Szörényi, T. Nepusz, and T. Vicsek, "Outdoor flocking and formation flight with autonomous aerial robots," in Proc. of 2014 IEEE/RSJ International Conference on Intelligent Robots and Systems, pp. 3866-3873, 2014. Article (CrossRef Link)

[24] A. Pospischil, "Mapping ad hoc communications network of a large number fixed-wing uav swarm," Naval Postgraduate School Monterey United States, 2017.

[25] A. Faust, I. Palunko, P. Cruz, R. Fierro, and L. Tapia, "Learning swing-free trajectories for UAVs with a suspended load," in Proc. of 2013 IEEE International Conference on Robotics and Automation, pp. 4902-4909, 2013. Article (CrossRef Link)

[26] H. Bou-Ammar, H. Voos, and W. Ertel, "Controller design for quadrotor uavs using reinforcement learning," in Proc. of 2010 IEEE International Conference on Control Applications, pp. 2130-2135, 2010. Article (CrossRef Link)

[27] S. R. B. dos Santos, C. L. Nascimento, and S. N. Givigi, "Design of attitude and path tracking controllers for quad-rotor robots using reinforcement learning," in Proc. of 2012 IEEE Aerospace Conference, pp. 1-16, 2012. Article (CrossRef Link)

[28] H. G. Tanner, A. Jadbabaie, and G. J. Pappas, "Flocking in fixed and switching networks," vol. 52, no. 5, pp. 863-868, 2007. Article (CrossRef Link)

[29] H. G. Tanner, A. Jadbabaie, and G. J. Pappas, "Stable flocking of mobile agents part I: dynamic topology," in Proc. of 42nd IEEE International Conference on Decision and Control (IEEE Cat. No. 03CH37475), vol. 2, pp. 2016-2021, 2003.

Article (CrossRef Link)

[30] C. Virágh, G. Vásárhelyi, N. Tarcai, T. Szörényi, G. Somorjai, T. Nepusz, and T. Vicsek, "Flocking algorithm for autonomous flying robots," Bioinspiration \& Biomimetics, vol. 9, no. 2, p. 025012, 2014. Article (CrossRef Link)

[31] J. Han, M. Li, and L. Guo, "Soft control on collective behavior of a group of autonomous agents by a shill agent," Journal of Systems Science and Complexity, vol. 19, no. 1, pp. 54-62, 2006. Article (CrossRef Link) 
[32] L. He, P. Bai, X. Liang, J. Zhang, and W. Wang, "Feedback formation control of UAV swarm with multiple implicit leaders," Aerospace Science and Technology, vol. 72, pp. 327334, 2018. Article (CrossRef Link)

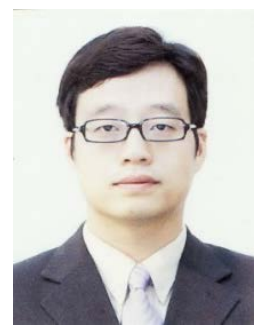

YoungHo Kwon received the B.S. degree in media communication from the Hanyang University, Seoul, Korea in 2003 and the Ph.D. degree in electrical \& computer engineering from the Hanyang University, Seoul, Korea in 2016. He was a lecturer in the department of Electrical and Electronic Engineering at the Hanyang University from 2012 to 2018. He is currently a postdoctoral researcher at Seoul Women's University. His current research interests include wireless communications, economic incentives for cooperation, social networking, and a vehicular ad-hoc network.

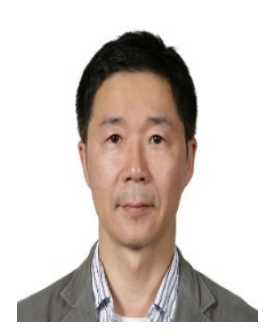

Jun Hwang is a professor in department of electrical \& computer engineering with Seoul Women's University. He has been a research professor in department of Electrical \& Computer Engineering, Georgia Institute of Technology. His research interests include primarily focused on the design and analysis of communication protocols for wired/wireless networks, Internet architectures, and mobile users, with an emphasis on distributed sensing, mobile applications, and multimedia entertainment. He is active in various technical program committees of the most prominent international conferences and is author of papers, which have been published in international conference proceedings and journals. was the general chair for the IFIP/IEEE Wireless Days 2010 conference, he was TPC chair of IEEE CCNC 2016, He is an academic chair of Korean Society for Internet Information (KSII) and executive director of Korea Cyber Guard Association. 